\title{
EEG data processing with neural network
}

\author{
Tamás Majoros \\ Intelligent Embedded Systems \\ Research Laboratory \\ Faculty of Informatics \\ University of Debrecen \\ Debrecen, Hungary \\ majoros.tamas@inf.unideb.hu
}

\author{
Balázs Ujvári \\ Department of Experimental Physics \\ Faculty of Science and Technology \\ University of Debrecen \\ Debrecen, Hungary \\ ujvarib@gmail.com
}

\author{
Stefan Oniga \\ (1) Faculty of Informatics \\ University of Debrecen, Hungary \\ (2) Intelligent Embedded Systems \\ Research Laboratory, Technical \\ University of Cluj-Napoca, North \\ University Centre of Baia Mare
}

\begin{abstract}
Machine-learning techniques allow to extract information from electroencephalographic (EEG) recordings of brain activity. By processing the measurement results of a publicly available EEG dataset, we were able to obtain information that could be used to train a feedforward neural network to classify two types of volunteer activities with high efficiency.
\end{abstract}

Keywords-neural network, classification, EEG, BCI, Fourier transform, ROOT, TMVA

\section{INTRODUCTION}

Electroencephalography (EEG) is a complex signal and can require several years of training to be correctly interpreted. Machine-learning techniques allow to extract information from EEG recordings of brain activity and therefore play a crucial role in several important EEG-based research and application areas. For example, machine-learning techniques are a central component of many EEG-based brain-computer interface (BCI) systems for clinical applications [1]. Such systems allow persons with severe paralysis to communicate [2], to draw pictures [3] and to control telepresence robots [4]. However, despite many examples of impressive progress, there is still room for considerable improvement with respect to the accuracy of information extraction from the EEG.

The goal of our work is to create a neural network and its hardware implementation that can recognize and evaluate brain waves, for example to help the elderly. Creating a database capable of training neural networks is cumbersome, requires many volunteers, advanced EEG sensors, data acquisition systems and requires months of work. One possible way to work around this problem is to use a publicly available database. Our idea is using a neural network trained on a large public database to interpret the output data of a similar device of our own.

\section{Physionet EEG DATABASE}

Recently, the availability of large EEG datasets and advances in machine learning have both led to the widespread use of machine learning architectures, especially in the analysis of EEG signals and in understanding the information it may contain for brain functionality. The robust automatic classification of these signals is an important step towards making the use of EEG more practical in many applications and less reliant on trained professionals [5].

Most of these datasets are recorded for evaluating some health-related issues, for example epileptic seizures. Other popular application is motor movement and imagery classification. One of the largest of these databases is the PhysioNet EEG database, which consists of over 1500 oneand two-minute EEG recordings, obtained from 109 volunteers. The volunteers performed six different tasks while 64-channel EEG were recorded using the BCI2000 system. Each subject performed 14 experimental runs: two one-minute baseline runs (one with eyes open, one with eyes closed), and three two-minute runs of each of the four following tasks:

- A target appears on either the left or the right side of the screen. The subject opens and closes the corresponding fist until the target disappears. Then the subject relaxes.

- A target appears on either the left or the right side of the screen. The subject imagines opening and closing the corresponding fist until the target disappears. Then the subject relaxes.

- A target appears on either the top or the bottom of the screen. The subject opens and closes either both fists (if the target is on top) or both feet (if the target is on the bottom) until the target disappears. Then the subject relaxes.

- A target appears on either the top or the bottom of the screen. The subject imagines opening and closing either both fists (if the target is on top) or both feet (if the target is on the bottom) until the target disappears. Then the subject relaxes [6].

The data are provided in EDF+ format, sampling frequency is 160 samples per second. The EEGs were recorded from 64 electrodes as per the international 10-10 system, excluding electrodes Nz, F9, F10, FT9, FT10, A1, A2, TP9, TP10, P9, and P10. The order of appearance of the electrodes in the data records is shown in Figure 1.

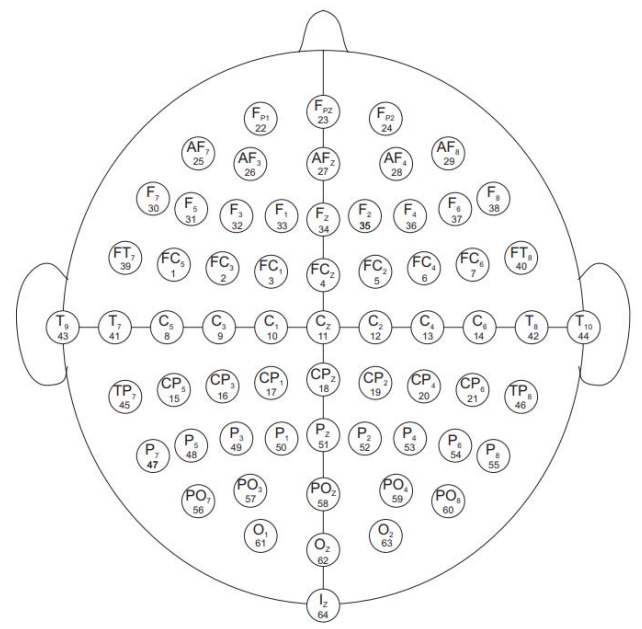

Fig. 1. Placement of 64 electrodes on head 


\section{SIGNAL PROCESSING}

In case of first two tasks (open and closed eyes), measurements were continuous for sixty seconds, whereas for motor movement and imagery activities, participants were given new tasks every four seconds. Accordingly, the sixtysecond measurements have been divided into four-second slots. Measurement data for the first four seconds of the first volunteer on the first channel is shown in Figure 2.

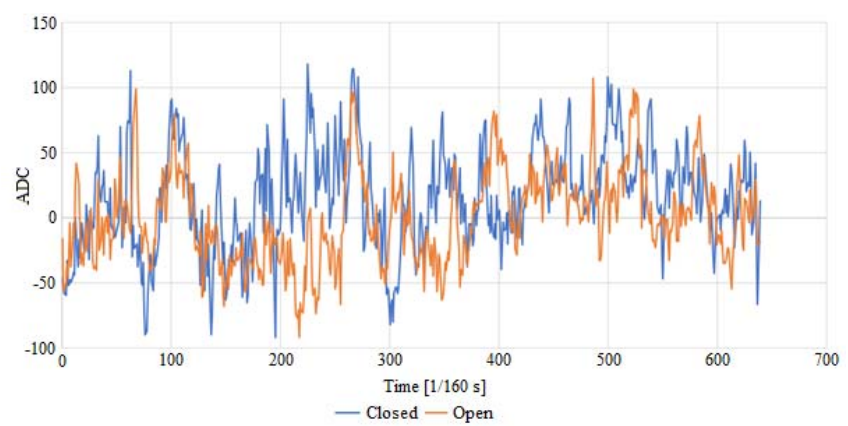

Fig. 2. Measurement data for the first four seconds of the first volunteer on the first channel

The time series stored in the database were then converted to frequency domain using Fourier transform. This was done for each channel, and the amplitudes against frequency and channel were plotted on a two-dimensional histogram (Figure 3).

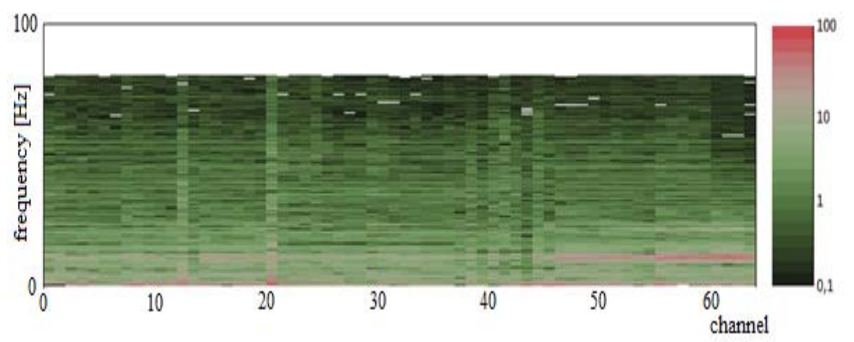

Fig. 3. Fourier transform of a four-second measurement

In order to distinguish between changes in cerebral activity during the two different tasks and independent cerebral activity that are otherwise present, histograms have been prepared for each volunteer showing the difference in amplitude for each channel and frequency. The results for the first two timeslots of one volunteer are shown in Figure 4 (open-closed) and Figure 5 (closed-open).

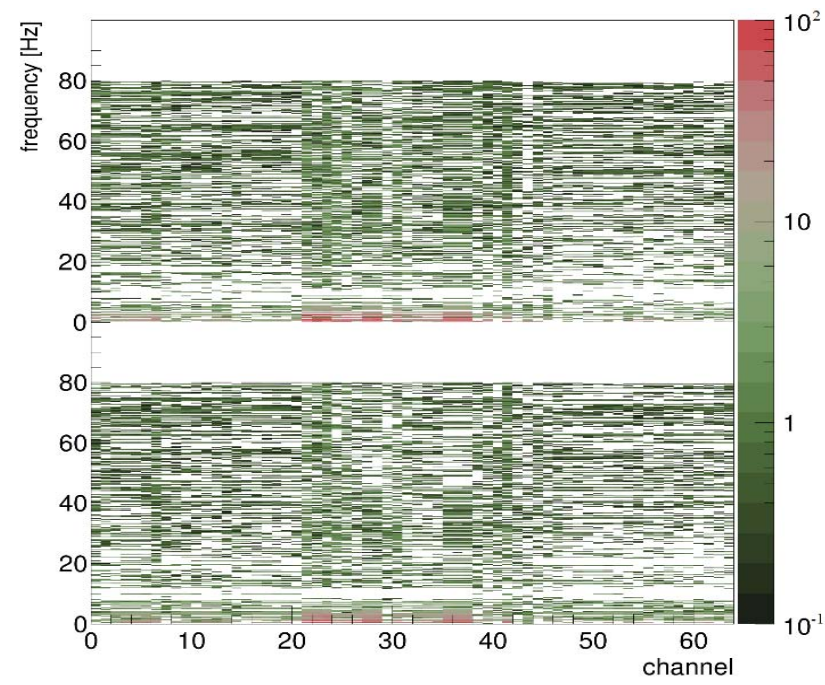

Fig. 4. Difference histograms, open-closed eyes, first two time slices

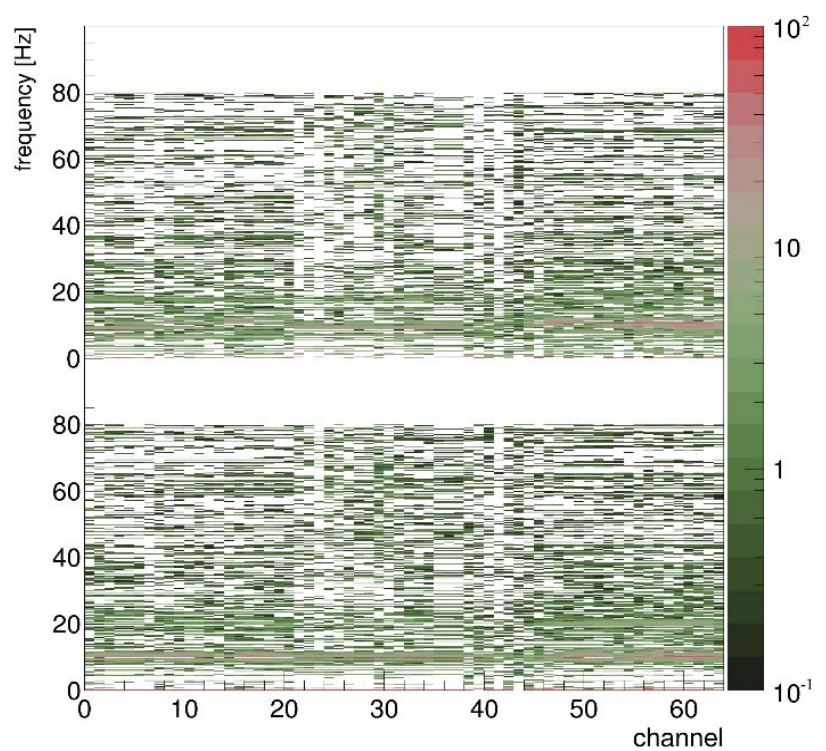

Fig. 5. Difference histograms, closed-open eyes, first two time slices

Figure 4 and 5 clearly show that there are several orders of magnitude differences in amplitudes for some channels and frequencies relative to noise. Gaussian curve fitting was used to investigate this. The fitting was performed separately for each channel, with a continuous shift of the fitting window along the frequency with different window sizes. In the case where the amplitude of the fitted function in a window under test exceeds a certain threshold, its expected value is displayed in the corresponding cell of a histogram as shown in Figure 6 (open-closed) and Figure 7 (closed-open).

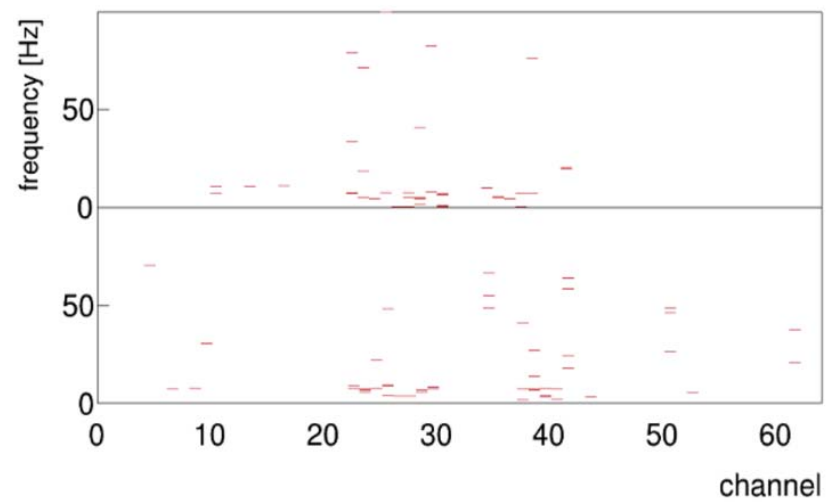

Fig. 6. Expected values of Gaussian functions with amplitude above $\mathrm{TH}=10$ threshold, open-closed eyes, $4 \mathrm{~Hz}$ fit window size

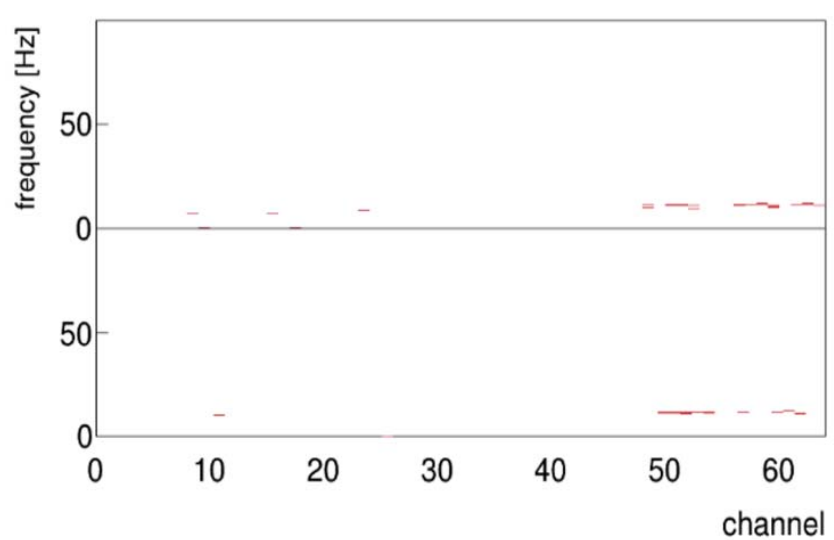

Fig. 7. Expected values of Gaussian functions with amplitude above $\mathrm{TH}=10$ threshold, closed-open eyes, $4 \mathrm{~Hz}$ fit window size 
The result of the function fitting shows that in the case of higher number channels, increased brain activity can be measured around the frequency of $10 \mathrm{~Hz}$. Based on the numbering shown in Figure 1, these channels (47-64) are located in the region of the occipital cortex. This observation is in agreement with the literature that the alpha wave correlates most closely with the closed-eyed but awake state [7]. The alpha wave is a base wave of 8 to $12 \mathrm{~Hz}$ with a higher amplitude above the occipital regions.

Taking a closer look, with 3D rendering, Figure 8 shows the alpha wave peak when the eyes are closed.

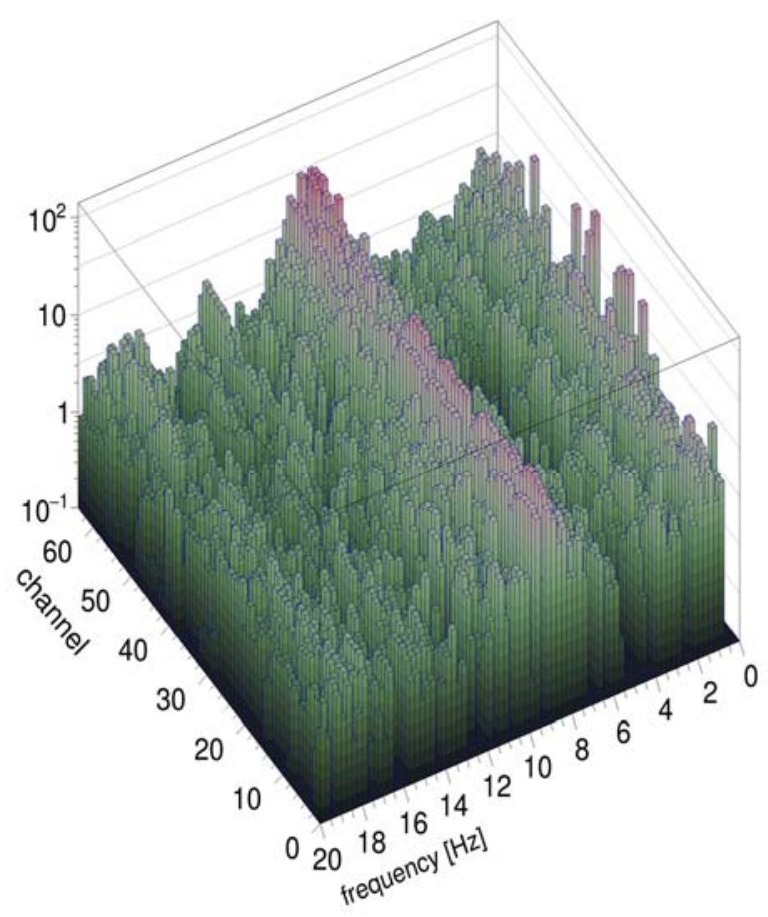

Fig. 8. Alpha wave, with higher amplitude at occipital region (closed eyes)

The reliability of the measurement results was investigated by various methods, which are not detailed due to extent, however, in case of many volunteers, problems were found that distort the final result.

\section{ROOT AND TMVA}

ROOT is an object-oriented program and library developed by CERN, which provides platform independent access to a computer's graphics subsystem and operating system using abstract layers. The packages provided by ROOT include histogramming, graphing, curve fitting, statistics tools, matrix algebra, standard mathematical functions, 3D visualizations, multivariate data analysis (e.g. using neural networks).

The Toolkit for Multivariate Analysis (TMVA) provides a ROOT-integrated environment for the processing, parallel evaluation and application of multivariate classification and regression techniques. All multivariate techniques in TMVA belong to the family of supervised learning algorithms, which make use of training events, for which the desired output is known, in order to determine the mapping function that either describes a decision boundary (classification) or an approximation of the underlying functional behavior defining the target value (regression). The software package provides training, testing, performance evaluation algorithms and visualization scripts.

TMultiLayerPerceptron is a class in the TMVA package. It describes a multilayer perceptron (MLP), which is a class of the feedforward artificial neural network. It contains facilities to train the network and to use the output. The input layer is made of inactive neurons (returning the normalized input), while hidden layers are made of neurons with an activation function, and output neurons are linear. The basic input is a training and a test event list. For classification jobs, a branch must contain the expected output. Different learning methods are available.

The most common algorithm for adjusting the weights that optimize the classification performance of a neural network is the so-called back propagation, which belongs to the family of supervised learning methods, we also used this. During the learning process the network is supplied with training events. For each training event the neural network output is computed and compared to the desired output ( 1 for open eyes, 0 for closed eyes). An error function measures the agreement of the network response to the desired one. The set of weights that minimizes the error function can be found using the method of gradient descent, provided that the neuron response function is differentiable with respect to the input weights. Starting from a random set of weights, the weights are updated by moving into the direction where error function decreases most rapidly [8].

\section{APPLICATION OF NEURAL NETWORK}

For frequency data, a resolution of $0.25 \mathrm{~Hz}$ is available. Since this would be an unreasonable number of inputs for the neural network, we have previously integrated the amplitudes per channel in the 8-12 Hz range (alpha waves). Inputs were data from electrodes in the occipital lobe, with four-second slices shifted second by second, from 50 volunteers, resulting in a total of 5700 data combinations. By random selection, half of this was used for training, the other half was used for testing only. The activation function of the neurons was of the sigmoid type, the network contained three hidden layers, with 3-3 neurons per layer, as shown in Figure 9. Type means activity type (open or closed eyes).

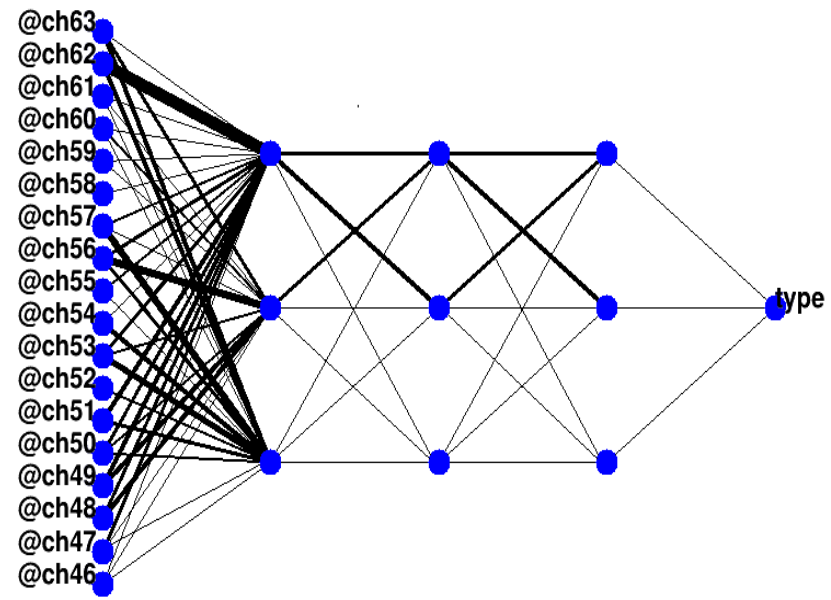

Fig. 9. Structure of the neural network 
The classification efficiency of the neural network was significantly impaired by data from erroneous measurements, however, we were able to obtain satisfactory results and to recognize with reasonable accuracy the two different states of the eye from the EEG data, as shown in Figure 10. Applying a cut of 0.5 to the output value, the neural network gave the correct classification result in 4690 cases out of 5700, which means a recognition accuracy of $82.28 \%$.

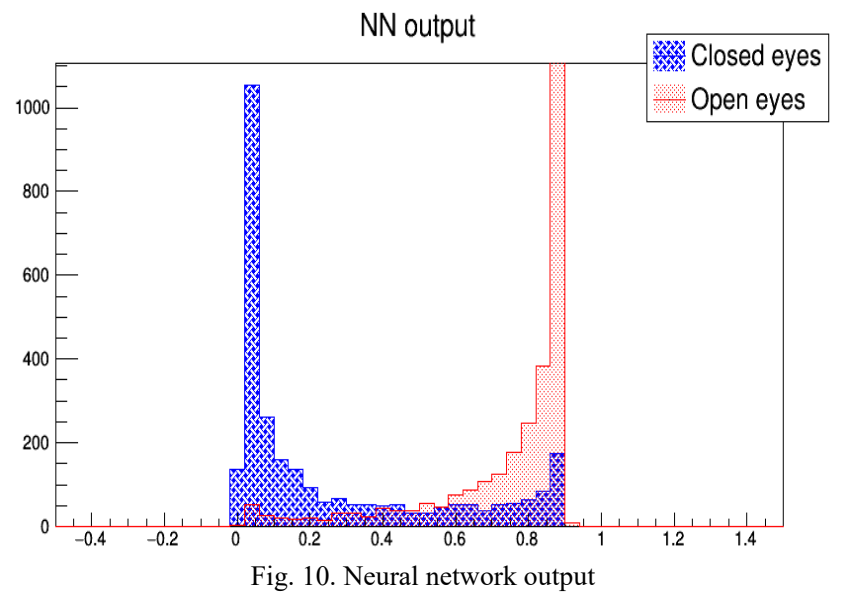

\section{ACKNOWLEDGMENT}

This work was supported by the construction EFOP3.6.3-VEKOP-16-2017-00002.

The project was co-financed by the Hungarian Government and the European Social Fund.

\section{REFERENCES}

[1] R. T. Schirrmeister, J. T. Springenberg, ..., T. Ball (2018): Deep learning with convolutional neural networks for brain mapping and decoding of movement-related information from the human EEG. arXiv:1703.05051v5

[2] Nijboer, F., Sellers, E. W., .., Kübler, A. (2008): A P300-based braincomputer interface for people with amyotrophic lateral sclerosis. Clincical Neurophysiology: Official Journal of the International Federation of Clinical Neurophysiology, 119(8):1909-1916.

[3] Munßinger, J. I., Halder, ..., Kubler, A. (2010). Brain Painting: First Evaluation of a New Brain-Computer Interface Application with ALSPatients and Healthy Volunteers. Frontiers in Neuroscience, 4.

[4] Tonin, L., Carlson, T., Leeb, R., Millán, J. d. R. (2011). Braincontrolled telepresence robot by motor-disabled people. In 2011 Annual International Conference of the IEEE Engineering in Medicine and Biology Society, pages 4227-4230.

[5] Craik, A., He, Y., Contreras-Vidal JL. (2019): Deep learning for electroencephalogram (EEG) classification tasks: a review. Journal of neural engineering. doi: 10.1088/1741-2552/ab0ab5

[6] Schalk, G., McFarland, D.J., Hinterberger, T., Birbaumer, N., Wolpaw, J.R. BCI2000: A General-Purpose Brain-Computer Interface (BCI) System. IEEE Transactions on Biomedical Engineering 51(6):10341043, 2004.

[7] M. Toscani, T. Marzi, S. Righi, M. P. Viggiano, S. Baldassi (2010): Alpha waves: a neural signature of visual suppression. Experimental brain researches. 207(3-4):213-9. doi: 10.1007/s00221-010-2444-7

[8] A. Hoecker, P. Speckmayer, J. Stelzer, J. Therhaag, E. von Toerne, H. Voss: TMVA 4 Users Guide

[9] J. Suto, S. Oniga, C. Lung, I. Orha, Comparison of offline and realtime human activity recognition results using machine learning techniques, Neural Computing and Applications, March 2018. https://doi.org/10.1007/s00521-018-3437-x 